\title{
Are defect models consistent with the entropy and specific heat of glass-formers?
}

\author{
Giulio Biroli \\ Service de Physique de Théorique, Orme des Merisiers, \\ CEA Saclay, 91191 Gif sur Yvette Cedex, France; \\ Jean-Philippe Bouchaud \\ Service de Physique de l'État Condensé, Orme des Merisiers, \\ CEA Saclay, 91191 Gif sur Yvette Cedex, France; \\ and Science 83 Finance, Capital Fund Management, 6-8 Bd Haussmann, 75009 Paris, France. \\ Gilles Tarjus \\ Laboratoire de Physique Théorique des Liquides, 4 Place Jussieu, 75231 Paris Cedex 05, France
}

(Dated: November 20, 2018)

\begin{abstract}
We show that point-like defect model of glasses cannot explain thermodynamic properties of glassformers, as for example the excess specific heat close to the glass transition, contrary to the claim of J.P. Garrahan, D. Chandler [Proc. Natl. Acad. Sci. 100, 9710 (2003)]. More general models and approaches in terms of extended defects are also discussed.
\end{abstract}

Kinetically constrained models (KCM) display trivial thermodynamics but non trivial dynamics [1, 2, 3]. They encapsulate in a specific way the 'free volume' idea according to which the slow dynamics of glasses is due to rare, localized mobility defects [4, 5]. For most KCMs, slow dynamics is indeed due to defect motions [3]. A remarkable aspect of these models is that although the dynamical rules are very simple and local, the emergent defect dynamics lead to highly non trivial physical behaviour. As a consequence KCMs provide a theoretical framework for understanding many of the remarkable dynamical properties of fragile glass-formers, such as superArrhenius relaxation time [3, 6, 7], dynamical heterogeneities [8, 9, 10, 11] and viscosity/diffusion decoupling [12. That these models may in fact describe quantitatively the physical properties of glasses has been strongly advocated in a series of papers by J.P. Garrahan and D. Chandler (GC) [9, 10], and investigated further in [13].

One of the major tenet of KCMs is the complete decoupling between dynamics and thermodynamics, at variance with the traditional view of Adam and Gibbs 14], and others 15, 16], in which the decrease of configurational entropy with temperature is the fundamental underlying mechanism for the viscous slowing down. It is therefore a priori surprising that KCMs could have anything sensible to say about the entropy and specific heat of real materials - even if these turn out to be acceptable models of their dynamics. Nevertheless, the claim made in [10] is that these models are also in quantitative agreement with the specific heat of these materials. More precisely, assuming a perfect gas of free defects, GC give a formula for the specific heat at fixed pressure per particle of the liquid (in excess of that of the solid), which we reproduce from ref. 10 to be:

$$
\Delta C_{p}(T)=k_{B}\left(\frac{J}{T}\right)^{2} c(T) \mathcal{N}+O\left(c(T)^{2}\right),
$$

where $\mathcal{N}$ is the number of physical molecules contributing to enthalpy fluctuations in a coarse-graining cell, $J$ is the enthalpy cost, in kelvins, for creating a mobile cell (remember that liquids are considered here at constant pressure, hence the importance of distinguishing between enthalpy and energy). The concentration of mobile cells $c$ is assumed to be given by $c(T) /(1-c(T))=A \exp (-J / T)$, where the numerical prefactor $A$ is argued by GC to be rather large: $\ln A$ is akin to an entropy gain $\Delta s_{0} / k_{B}$ associated with the creation of a mobile cell. Note that there is a small difference between the specific heat in excess to that of the glass (considered in [10]) and that in excess of the crystal; this difference is however irrelevant for the present discussion.

In the following we first adress the results of [10] showing that even if the value of $\Delta C_{p}$ at $T_{g}$ seems to match experimental values, the temperature dependence of $\Delta C_{p}$ predicted by Eq. (11) is in total disagreement with experimental values. We then discuss in detail the derivation of $\Delta C_{p}(T)$ and the physical assumptions behind Eq. (1). This derivation leads to a formula different from Eq. (1) and makes clear that the defect contribution to $\Delta C_{p}(T)$ is in fact completely irrelevant close to the glass transition. In order to avoid a contradiction, one has to assume that the main contribution to both the entropy and the specific heat comes from the immobile regions. Although this is most reasonable physically, it implies that the models considered by GC are incapable of reproducing the thermodynamics of fragile glasses (and actually neither of strong glasses).

A clean way to test Eq. (1), that gets rid of any ambiguity in prefactors (we in fact disagree with the prefactor $\mathcal{N}$ in Eq. (1), see below), is to rewrite it as:

$$
\frac{\Delta C_{p}(T)}{\Delta C_{p}\left(T_{g}\right)} \approx t^{-2} \exp \left(\frac{J}{T_{g}} \frac{t-1}{t}\right) \quad t=\frac{T}{T_{g}} .
$$

This formula is expected to be valid for small $c \approx$ 
$A \exp (-J / T)$, where the description of glassy dynamics in terms of rare, dilute, defects could make sense - corresponding to a large enthalpy of creation for mobility defects, $J / T_{g} \gg 1$.

Using viscosity data at fixed (atmospheric) pressure, GC are able to estimate the value of $J / T_{g}$, found to be equal to 16.7 for 3 -bromopentane (3BP) and 26.7 for ortho-terphenyl (OTP), indeed quite large compared to unity. Fig. 1, on the other hand, gives a plot of $\Delta C_{p}(T) / \Delta C_{p}\left(T_{g}\right)$, obtained from Eq. (2), as a function of $T / T_{g}$ for $J / T_{g}=10,20,30$ and compares it to the experimental values for 3BP [17] and OTP [18]. (Note the $\log$ scale on the $y$-axis.) It is clear that Eq. (2) is totally incompatible with the data when $J / T_{g}$ is large: it varies much too fast with temperature, and increases with $T$ instead of decreasing (a similar criticism has been made by C. Angell and coworkers 19] for excitation models).

An acceptable fit of the data with Eq. (2) requires $J / T_{g} \approx 1.3$, meaning that within this description the density of defects is not small at all - but this of course is then incompatible with viscosity data. For consistency, Eq. (11) cannot be used for temperatures such that $c \simeq 1$; GC restrict their analysis to a temperature range between $T_{g}$ and $T_{1 / 2}$, where $T_{1 / 2}$ is defined as the temperature at which the logarithm of the viscosity expressed in Poise, or of the relaxation time expressed in picoseconds is half its value at $T_{g}$ (see Fig. 1). One can clearly see that, even in this restricted interval of temperature close to $T_{g}$ (for which $c$ is of the order or less than $10^{-1}[10]$ ), the theoretical prediction is in complete disagreement with the experimental data.

The previous discussion suggests that the thermodynamic predictions of the models considered in [10] are incompatible with experiments. As we discuss below, there is in fact no real contradiction: the order of magnitude of the defect contribution to the specific heat is simply much smaller than the experimental value between $T_{g}$ and $T_{1 / 2}$, which is instead dominated by the thermodynamic properties of immobile regions. In the models considered in [9, 10], however, the immobile regions give a trivial contribution to thermodynamics properties. In order to be consistent, one should assume that these regions dominate the thermodynamics but are irrelevant dynamically.

Let us now reconsider in detail the derivation of Eq. (11) taking explicitely into account that experimentally both the specific heat and the excess entropy are found to be of order $k_{B}$ per particle or larger at the glass temperature indeed a very important "stylized fact" of fragile glasses. An immediate consequence of this experimental fact is that the excess entropy of the liquid cannot be due to point-like defects only: since these must be dilute to lead to large relaxation times, their contribution to the excess entropy must be small. Within a simple coarse-grained defect description, the total excess entropy per cell (with

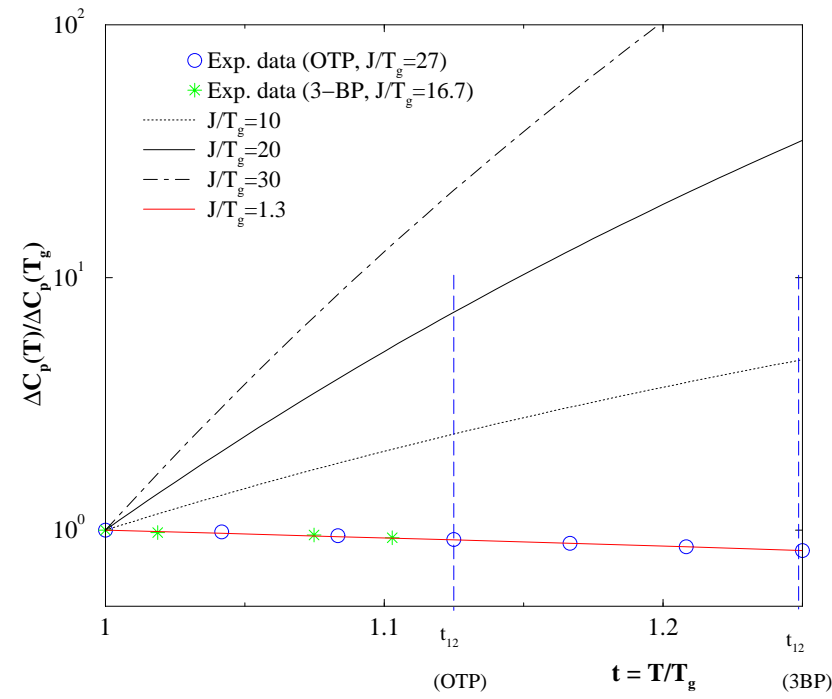

FIG. 1: Normalized excess specific heat as given by GC's formula, Eqs. (1) and (2), for $J / T_{g}=10,20,30$, compared with the experimental data for OTP and 3-BP, for which GC estimate, from viscosity data, $J / T_{g}=26.7$ and 16.7 , respectively. Note the $\log$ scale on the $y$-axis. The two vertical lines correspond to $T_{1 / 2}$ defined as the temperature at which the logarithm of the viscosity expressed in Poise, or of the relaxation time expressed in picoseconds is half its value at $T_{g}$.

respect to the crystal), $\Delta s$, is:

$$
\Delta s=-k_{B}[c \ln c+(1-c) \ln (1-c)]+c \Delta s_{0}+\Delta s_{c},
$$

where the first term corresponds to the entropy of the ideal gas of defects, the second term to the entropy difference between mobile and immobile cells (see above), and the last term is the configurational entropy associated with frozen cells 21]. In order to get the experimental order of magnitude of $\Delta s$ at $T_{g}$ and at the same time have $\Delta C_{p}$ dominated by defect properties, one must assume that (i) $\Delta s_{c}$ is of order $\mathcal{N} k_{B}$, hence much larger than the defect entropy itself; (ii) $\Delta s_{c}$ must then be temperature independent, otherwise it would give the leading contribution to $\Delta C_{p}$. Although large, the excess entropy $\Delta s_{c}$ should not play any physical role in the slowing down of the material in this picture (in strong contrast with the view expressed in [14, 15, 16]). Therefore, the excess specific heat at constant pressure per cell, $\Delta c_{p}(T)=T d \Delta s(T) / d T$, is given by:

$$
\Delta c_{p}(T)=k_{B} \frac{J}{T} T \frac{d c(T)}{d T} \approx k_{B}(J / T)^{2} c(T),
$$

where we have used $c(T) \approx \exp \left(\Delta s_{0}-J / T\right)$, valid in the limit $c \ll 1$. Note that the functional form of $c(T)$ cannot be derived within the model and it is in principle obtained from the coarse-grained procedure that should map glass-forming liquids to KCMs. Since until now no such procedure has been developed one has to postulate 
an a priori functional form $c(T)$. The simple physical assumptions described above correspond to the one assumed in 10. However, we will show below that our conclusion is indeed independent of the form of $c(T)$. We have obtained a specific heat per cell given by $k_{B}(J / T)^{2} c$, and therefore a specific heat per molecule given by:

$$
\Delta C_{p}(T) \approx k_{B}\left(\frac{J}{T}\right)^{2} c(T) \mathcal{N}^{-1},
$$

a factor $\mathcal{N}^{2}$ smaller than Eq. (11). The same expression is obtained by looking directly at the enthalpy fluctuations 22]. Taking very favorable values for the parameters $c=$ $0.01, J / T_{g}=30$ and $\mathcal{N}=10$ leads to $\Delta C_{p}\left(T_{g}\right) \approx 0.1 k_{B}$, already a factor 100 too small compared to experimental values $\left(9 k_{B}\right.$ for 3 -BP, $13 k_{B}$ for OTP). The only way to avoid a contradiction is that the temperature dependence of $\Delta s_{c}$ is strong, and that in fact all the thermodynamics is contained in that very contribution.

The above conclusion is based on comparing the experimental specific heat to that predicted by defects only. A more general argument (see also [16, 20]), independent of the shape of $c(T)$, relies on the entropy change between - say $-T_{g}$ and $T_{1 / 2}$, which is experimentally found to found to be of order $k_{B}\left(\approx 4 k_{B}\right.$ for OTP). Thus, not only one finds that $\Delta s_{C}(T)$ has to be much larger than $\left(-k_{B}[c \ln c+(1-c) \ln (1-c)]+c \Delta s_{0}\right)$ at $T_{g}$, as discussed previously, but also that $\Delta s_{c}(T)$ has to provide the leading contribution between $T_{g}$ and $T_{1 / 2}$ since in this regime the ideal gas entropy of defects 23 is negligible [10]. As a consequence, using the relationship $\Delta c_{p}(T)=T d \Delta s(T) / d T$, one finds again that the main contribution to $\Delta C_{p}$ comes from the immobile regions close to $T_{g}$. Note that this result is independent of the functional form $c(T)$, as long as defects are dilute, $c \ll 1$, a necessary condition for the GC description to be useful 24.

So, are all KCMs (or more generally defect models) doomed to fail in describing thermodynamics? The point of view advocated in [9, 10] is that after having appropriately coarse-grained time and space a liquid can be strictly speaking considered as a KCM with trivial thermodynamics and simple kinetic constraints that induce slow dynamics. As we have shown, this line of thought is clearly insufficient to recover thermodynamics. Another point of view (see for example [2, 6]) is to only assume that the effective dynamics for a lattice model of a glass-forming liquids is characterized by some kinetic constraints. In this case the trivial thermodynamics is not necessary but just a choice of simplicity. A quantitative model (arising from a real mapping from liquid dynamics) would also contain effective interactions between particles and would therefore lead to non-trivial thermodynamics. One important fact remains though: even if the thermodynamics can be non trivial, it is only an indirect cause of slow dynamics, through the temperature dependence of the defect density, and not a driving cause of the slowing down. In our view, any eligible theory of the glassy state has to produce a convincing explanation of the remarkable connection between thermodynamics and dynamics, more precisely between the configurational entropy and the relaxation time [26]. At the moment this seems to be an important missing piece in KCMs. Hopefully, future works on KCMs and their possible generalisations will unveil whether or not some of these models can pass this important test. A way to increase the entropy contribution of mobility defects and find a relationship between dynamics and thermodynamics seems to be through extended defects. This is the path followed (using different arguments) in 14, 15, 16, 27, 28, 29] (see also 30, 31] for quantitative computation of the excess configurational entropy in model systems). In all these scenarii, the presence of mobility regions, that are typically interfaces (e.g. domain walls), is driven by a thermodynamic mechanism with a (possibly avoided) critical point.

We thank C. Alba-Simionesco, L. Berthier and D.R. Reichman for interesting discussions. GB is partially supported by the European Community's Human Potential Programme contracts HPRN-CT-2002-00307 (DYGLAGEMEM).

\section{Addendum}

In the following reply, Chandler and Garrahan have amended their original model which now allows for two different species of excited cells - most of them are in this new version immobile, and very few are mobile. Even though the microscopic justification of this ad hoc assumption is unclear, the new fitting parameter, the ratio of excited mobile to excited immobile cells, indeed allows one to obtain both a large specific heat jump and a large relaxation time. In such a framework, however, the strong connection between thermodynamical and dynamical fragility, which we emphasized above, becomes completely out of reach or very artificial (thermodynamics is dominated by immobile excited cells that are irrelevant as far as dynamics is concerned). We also note that despite the introduction of another fitting parameter [the temperature dependence of the ratio of excited mobile to excited immobile cells] the theory proposed by Chandler and Garrahan still leads to a specific heat which has the wrong temperature dependence - note that the scale of the y-axis in their Fig. 1 makes it hard to see that their prediction for the excess of specific heat is in fact increasing with temperature, in contradiction with experiments that clearly show an opposite trend. It might be possible, by introducing additional assumptions and fitting parameters, to obtain yet another version of Chandler and Garrahan's model that "accounts for" these basic experimental facts. However, all this is at the expense of the simplicity, the predictive power and, more importantly, the very meaning of the underlying physical picture. 
[1] G.H. Fredrickson and H.C. Andersen, Phys. Rev. Lett. 53, 1244 (1984); J. Chem. Phys. 83,5822 (1985). G.H. Fredrickson and S.A. Brawer J. Chem. Phys. 84, 3351 (1986).

[2] W. Kob and H.C. Andersen, Phys. Rev. E 48 (1993) 4364.

[3] for a review, see F. Ritort, P. Sollich, Adv. Phys. 52, 219 (2003).

[4] M.H. Cohen and G.S. Grest, Phys. Rev B 20, 1077 (1979); 214113 (1980).

[5] S.H. Glarum, J. Chem. Phys. 33, 639 (1960)

[6] C. Toninelli, G. Biroli, and D. S. Fisher Phys. Rev. Lett. 92, 185504 (2004) and cond-mat/0410647

[7] P. Sollich , M.R. Evans, Phys. Rev. Lett 83, 3238 (1999).

[8] P. Harrowell Phys. Rev. E 48, 4359 (1993). M.M. Hurley, P. Harrowell, Phys. Rev. E 521694 (1995) and refs therein.

[9] J.P. Garrahan, D. Chandler, Phys. Rev. Lett. 89, 035704 (2002).

[10] J.P. Garrahan, D. Chandler, Proc. Natl. Acad. Sci. 100, 9710 (2003).

[11] S. Franz, R. Mulet and G. Parisi, Phys. Rev. E 65, (2002) 021506.

[12] Y. Jung, J.P. Garrahan, D. Chandler, Phys. Rev. E, 69, 061205 (2004). Length scale for the onset of Fickian diffusion in supercooled liquids, L. Berthier, D. Chandler, J. P. Garrahan, cond-mat/0409428

[13] L. Berthier, J.P. Garrahan, Phys. Rev. E 68, 041201 (2003); S. Whitelam, L. Berthier, J.P. Garrahan, Phys. Rev. Lett. 92, 185705 (2004); L. Berthier, J.P. Garrahan, Numerical study of a fragile three-dimensional kinetically constrained model, cond-mat/0410076

[14] G. Adam, J. H. Gibbs, J. Chem. Phys. 43139 (1958).

[15] T. Kirkpatrick, P. Wolynes, Phys. Rev. B 36, 8552 (1987); T. R. Kirkpatrick, D. Thirumalai, P. G. Wolynes, Phys. Rev. A 40 (1989) 1045; X. Xia, P. G. Wolynes, Proc. Nat. Acad. Sci. 97, 2990 (2000), Phys. Rev. Lett 86, 5526 (2001).

[16] J. P. Bouchaud, G. Biroli, J. Chem. Phys. 121, 7347 (2004)

[17] S. Takahara, O. Yamamuro, T. Matsuo, J. Phys. Chem. 99 (1995) 9589.

[18] S.S. Chang and A.B. Bestbul, J. Chem. Phys. 56 (1972) 503.
[19] R. Moynihan, C. A. Angell, J. Non. Cryst. Solids 274 , 131 (2000); C. A. Angell, J. Phys. Cond. Matter 12, 6463 (2000).

[20] V. Lubchenko and P.G. Wolynes, J. Chem. Phys. 121, 2852 (2004).

[21] Even if the difference between the heat capacities of the glass and the crystal is small, the entropy of the glass, considered as a "frozen liquid", is significantly higher than that of the crystal down to $0 \mathrm{~K}$.

[22] A very direct way to obtain Eq. (5) is writing the enthalpy per particle due to mobile cells $H=J c(T) / \mathcal{N}$ and using the thermodynamic relation $\Delta C_{p}(T)=d H / d T$.

[23] Even if there was a static interaction between defects, the entropy contribution cannot be larger than the ideal gas one.

[24] This in principle does not directly apply to strongly cooperative defect models. Their structural relaxation timescale (and inverse of the defect density) can increase extremely fast when the temperature decreases or the particle density increases [2, 3, [6]. But at the same time their entropy decreases mildly and smoothly in such a way that it is not necessary to have a very small entropy in order to have a very large relaxation time [25].

[25] M. Sellitto, J. Phys. Condens. Matter 12 (2000) 6477.

[26] C. A. Angell, Jour. Res. NIST, 102171 (1997); R. Richert and C. A. Angell, J. Chem. Phys. 1089016 (1998).

[27] S. Sachdev and D. R. Nelson, Phys. Rev. Lett. 53, 1947 (1984); Phys. Rev. B 32, 1480 (1985). D. R. Nelson and F. Spaepen, Solid State Phys. 42, 1 (1989).

[28] D. Kivelson, S. A. Kivelson, X.-L. Zhao, Z. Nussinov, and G. Tarjus, Physica A 219, 27 (1995); G. Tarjus and D. Kivelson, J. Chem. Phys. 103, 3071 (1995); ibid J. Chem. Phys. 109, 5481 (1998); D. Kivelson and G. Tarjus, Phil. Mag. B77, 245 (1998);

[29] V. Lubchenko and P.G. Wolynes, J. Chem. Phys. 119 9088 (2003), J. Chem. Phys. 121, 2852 (2004), Phys. Rev. Lett. 87 , 195901 (2001); X. Xia and P.G. Wolynes PNAS 972990 (2000) and refs. therein.

[30] B. Coluzzi, M. Mézard, G. Parisi, P. Verrocchio, J.Chem.Phys. 111, 9039 (1999).

[31] K.-K. Loh, K. Kawasaki, A. R. Bishop, T. Lookman, A. Saxena, J. Schmalian, Z. Nussinov, Glassy behavior in systems with Kac-type step-function interaction, cond-mat/0206494 